\title{
Morbidity Prevalence Rate of Kawasaki Disease Assessed by Single Cross-Sectional History-Taking
}

\author{
Taeko Tsuji, ${ }^{1} \mathrm{MD}$, Jun-ichi SuzuKI, ${ }^{1} \mathrm{MD}$, Ryoichi Shimamoto, ${ }^{1} \mathrm{MD}$, \\ Tadashi YAmAZAKi, ${ }^{1}$ MD, Yuki Oномото, ${ }^{1}$ MD, Kuniaki IwASAwa, ${ }^{1}$ MD, \\ and Ryozo NAGAI, ${ }^{1} \mathrm{MD}$
}

\section{SUMMARY}

The need for long-term follow-up in Kawasaki disease is poorly recognized although cardiac sudden death attacks asymptomatic young people with past illness after a long latent period. Therefore, in order to prevent cardiac disasters, high risk groups should be identified and the prevalence rate of the disease should be determined for crisis management.

A total of 9,965 consecutive freshmen at the University of Tokyo were the subject of a questionnaire. Their parents/guardians who were briefed on the diagnostic criteria of the acute phase of Kawasaki disease actually completed the questionnaire. Students with a positive diagnosis underwent rest and exercise-stress electrocardiography and routine echocardiography.

The overall prevalence rate was $0.57 \%$. The rate in males $(0.63 \%)$ was greater than that in females $(0.32 \%)(P<0.05)$. Electrocardiography and routine echocardiography identified no indices specific to a past illness of Kawasaki disease.

The prevalence rate indicated that about 6 in 1000 students were high risk students who needed special care while at university. Since there are few symptoms and no signs indicating a past illness of Kawasaki disease, intensive history-taking from parents/guardians who are familiar with their acute symptoms during childhood is required in order to identify those at high risk of a coronary event. (Int Heart J 2007; 48: 615-621)

Key words: Kawasaki disease, Prevalence rate, Cardiac disaster, High risk

\begin{abstract}
AsYMPTOMATIC teenagers and young adults without coronary risk factors can be suddenly killed by ischemic cardiac attacks. Postmortem examination in these cases sometimes demonstrates no underlying disorders except for coronary arterial lesions caused by an old specific disease. ${ }^{1-3)}$ Kawasaki disease, an acute febrile disease sometimes complicated with coronary artery inflammation, affects children less than 5 years of age ${ }^{4,5}$ and leads to disastrous outcomes after a long latent period. Because the prognosis of the acute febrile phase is favorable itself,

From the ${ }^{1}$ Department of Cardiovascular Medicine, Faculty of Medicine and Graduate School of Medicine, University of Tokyo, Tokyo, Japan.

Address for correspondence: Jun-ichi Suzuki, MD, Laboratory 213, Department of Cardiovascular Medicine, Faculty of Medicine and Graduate School of Medicine, University of Tokyo, 7-3-1 Hongo, Bunkyo-ku, Tokyo 113-8655, Japan.

Received for publication May 28, 2007.

Revised and accepted August 9, 2007.


and the patients are often asymptomatic until the sudden onset of coronary events, their parents have no idea of the need for long-term medical follow-up. Pediatricians have not been emphasizing the complex natural history of a coronary aneurysm, ie, normal coronary findings on morphological examinations performed in the chronic phase do not guarantee a future free from coronary attacks. Normal morphology confirmed by scrutiny not in the chronic phase but in the acute febrile phase is required to rule out the false normal coronary findings in the process of development from coronary dilatation in the acute phase to coronary stenosis in the chronic phase. ${ }^{6-8)}$

Due to the lack of symptoms and poor recognition of the necessity for intensive long-term follow-up of Kawasaki disease, it is necessary to establish specific and objective criteria by routine examination to identify high risk patients with a history of the illness. Therefore, the current study was designed in order to clarify whether electrocardiography and/or echocardiography have the capability to detect findings specific to Kawasaki disease at the chronic phase and an intensive history-taking from the parents or guardians of consecutive university freshmen was conducted to estimate the rate of prevalence of Kawasaki disease.

\section{MeTHODS}

Study population: The questionnaire on Kawasaki disease was conducted as a part of the health check of freshmen upon their entrance into the University of Tokyo. The study population consisted of 9,965 (male: 8,073 , female: 1,892) consecutive students who entered the University from 2001 through 2003 . The only requirement for admission is academic ability. The mean age of the students at the time the questionnaire was conducted was $18.4 \pm 1.5$ years.

Diagnosis of Kawasaki disease: All questions on the questionnaire form were filled out by the freshmen with their parents or guardians who knew their childhood medical history. On the basis of data obtained by the questionnaire, a past history of Kawasaki disease was established when the students had a history of high fever with a duration of 5 or more days accompanied by at least 4 of the following 5 signs in the acute febrile phase of the disease. The diagnostic signs include 1) erythema and desquamation in the fingertips, 2) polymorphous exanthema, 3) bilateral conjunctivitis, 4) erythema and cracking of the lips and oral cavity, and 5) cervical lymphadenopathy. A past history was also established in students with fever and 3 of the 5 signs when coronary arterial disease was documented with morphological examinations in the acute phase of the disease..$^{9,10}$ )

Prior to replying to the questionnaire, background medical information on Kawasaki disease, such as the symptoms, diagnostic criteria, and prognosis was given to all students and their parents and/or guardians. All students in whom a 
past history was confirmed by the current questionnaire underwent rest and exercise stress electrocardiography, and echocardiography.

Data analysis and statistical analysis: The frequency of each clinical sign was calculated for the 5 diagnostic signs in students with a confirmed diagnosis. ${ }^{9,10)}$

Asymptomatic students whose age and gender matched those of students with a past history of Kawasaki disease also underwent rest electrocardiography and routine echocardiography as a control group. The population size of the control group was also matched with that of the disease group. The controls had no past history concerning the cardiovascular system. Abnormal rhythms, conduction disturbances, abnormal Q waves, abnormalities of ST segments, abnormal T waves, and negative $U$ waves were examined on electrocardiograms. Existence of hypertrophy, wall motion abnormality of the left ventricle, and valvular dysfunction was examined by two-dimensional and color Doppler echocardiography. End-diastolic dimension and end-systolic dimension of the left ventricle were measured with M-mode echocardiography. In the current study, morphological examination of the coronary arteries was not routinely performed by echocardiography because of limited examination time.

Exercise induced symptoms, ST-T changes, and arrhythmias were evaluated by exercise stress electrocardiography.

All values are expressed as the mean \pm SD. Differences between the two groups were tested by either the unpaired Student's $t$-test or chi-square test. A $P$ value less than 0.05 indicated statistical significance.

\section{RESULTS}

Summary of questionnaire: A past history of Kawasaki disease was confirmed in 51 male students and 6 female students. The morbidity prevalence rate was $0.63 \%$ for male students and $0.32 \%$ for female students, and $0.57 \%$ overall. The prevalence for male students was statistically greater than that for female students $(P<0.05)$.

Table I. Frequency of Diagnostic Signs in Past Illness, the University of Tokyo, Japan, 2001-2003

\begin{tabular}{lcr}
\hline Diagnostic signs & Number of students affected & $(\%)$ \\
\hline Fever of at least 5 days' duration & 57 & $(100.0)$ \\
Change in Extremities & 56 & $(98.2)$ \\
Polymorphous exanthema & 53 & $(93.0)$ \\
Bilateral conjunctival infection & 54 & $(94.7)$ \\
Change in the lips and oral cavity & 57 & $(100.0)$ \\
Cervical lymphadenopathy & 47 & $(82.5)$ \\
\hline
\end{tabular}


Table II. Abnormal Findings Among the Students, the University of Tokyo, Japan, 2001-2003

\begin{tabular}{lccc}
\hline & Disease Group & Control Group & $P$ value \\
\hline ECG findings & & & \\
ST-T changes (\%) & 0.0 & 0.0 & \\
Abnormal Q wave (\%) & 1.8 & 0.0 & 0.3 \\
Echocardiographic findings & & & \\
Dd* (mm) & 47.6 & 47.2 & 0.6 \\
Ds* (mm) & 28.8 & 29.1 & 0.7 \\
Asynergy of LV* $(\%)$ & 0.0 & 0.0 & \\
\hline
\end{tabular}

* Dd indicates diastolic dimension; Ds, systolic dimension; and LV, left ventricle.

The frequency of each diagnostic sign in students with a confirmed diagnosis is summarized in Table I.

Electrocardiographic and echocardiographic findings: Table II presents a summary of the electrocardiographic and echocardiographic findings. There were no differences in the frequency of abnormal findings in either the electrocardiograms or echocardiograms.

Exercise stress electrocardiography: No symptoms such as chest pain or chest oppression during exercise were observed. There were no ST-T changes induced by exercise in 57 students. Isolated ventricular premature beats were observed in 3 students.

\section{DiscuSsiON}

Kawasaki disease in the acute phase with a favorable prognosis can be spontaneously cured, and therefore the diagnosis may not have always been established in the affected children by their pediatrician. Moreover, a spontaneous declaration of past illness by children and/or their guardians is unreliable because there are few symptoms in the chronic phase ${ }^{11-13)}$ and their poor awareness of the need for medical long-term follow-up. Accordingly, an intensive history-taking of the child's medical condition in the acute febrile phase by interviewing their parents who are briefed on the characteristics of Kawasaki disease is needed in order to obtain reliable information on the past history of the child as it related to Kawasaki disease.

No child contracts Kawasaki disease after the age of 10, and therefore, the sum of each yearly morbidity incidence rate from the age of zero through 9 in a single common study population represents the "morbidity prevalence rate" of Kawasaki disease. ${ }^{14-17)} \mathrm{A}$ few study has reported ${ }^{18)}$ the morbidity prevalence rate 
of Kawasaki disease because it takes a long time to sum up all of the incidence rates. Fortunately, because febrile diseases seldom affect children older than 10, a cross-sectional surveillance of the frequency rate of subjects who have a past history of Kawasaki disease after the age of 10 can provide the morbidity prevalence rate for Kawasaki disease. In the current study, the childhood illnesses of 9,965 consecutive freshmen entering the University of Tokyo were determined by conducting an intensive history-taking interview of their parents. As stated above, the frequency rate of the "past illness" of Kawasaki disease surveyed in this manner can be substituted for the "prevalence rate" of Kawasaki disease. In the current study, information on the diagnostic criteria was given to both students and their parents/guardians prior to data acquisition at the compulsory health check-up conducted in all consecutive freshmen when entering Tokyo University.

In conclusion, because no specific findings related to a past history of Kawasaki disease detected by clinical routine examinations at the chronic phase of Kawasaki disease have been reported ${ }^{19-21)}$ and the spontaneous declaration of Kawasaki disease in the past from students and their guardians is unreliable, intensive history-taking should be undertaken in parents or guardians who have been appropriately briefed on the disease in order to avoid overlooking students who are at high risk of sudden cardiac death. The morbidity prevalence rate of Kawasaki disease estimated as the frequency of a past history of the disease in the current study suggests that about 1 out of 200 asymptomatic students may have had the disease, and that this should be recognized in order to prevent possible cardiac disasters. 


\section{APPENDIX \\ Questionnaire for Kawasaki Disease \\ Freshmen's Health Check at the University of Tokyo}

Kawasaki disease is an acute febrile disease including inflammation of the coronary arteries in children less than 5 years of age. After recovery from the acute phase, the disease is sometimes associated with lethal complications following a long latent period that may result in a coronary disaster such as cardiac sudden death in university students, especially during physical exercise.

To prevent such a disaster and identify high-risk students, please answer the following questions with your parent(s)/guardian(s) who is/are familiar with your childhood illnesses.

Query-1 Have you ever been diagnosed with Kawasaki disease? (yes, no) If yes, please identify the institute where the diagnosis was made.

Name of Institute:

Query-2 Have you ever undergone cardiac catheterization? (yes, no)

Query-3 Did you undergo treadmill exercise stress electrocardiography within the last year?

(yes, no)

Query-4 Have you ever been diagnosed with a coronary aneurysm(s)? (yes, no) Query-5 Do you have a past history of high fever lasting 5 or more days?

If yes, was the fever accompanied by any of the following signs?

1) Swelling of lymph nodes at the cervix (cervical lymphadenopathy)

2) Bilateral reddish conjunctivae (conjunctivitis) (yes, no)

3) Erythema and/or desquamation in the fingertips (yes, no)

4) Erythema and cracking of the lips and oral cavity (yes, no)

5) Polymorphous exanthema (yes, no) (yes, no)

Query-6 Please give the name of the parent(s)/guardian(s) with whom you completed this questionnaire.

Relative(s): 


\section{REFERENCES}

1. Rozin L, Koehler SA, Shakir A, Ladham S, Wecht CH. Kawasaki disease: a review of pathologic features of stage IV disease and two cases of sudden death among asymptotic young adults. Am J Forensic Med Pathol 2003; 24: 45-50.

2. Bartoloni G, Salvatrice DM, Carlo R. Sudden death in a 21-year-old man caused by thrombosed coronary aneurysm: late sequelae or a very late onset of Kawasaki disease? Cardiovasc Pathol 2002; 11: 318-21.

3. Maresi E, Passantino R, Midulla R, et al. Sudden infant death caused by a ruptured coronary aneurysm during acute phase of atypical Kawasaki disease. Human Pathology 2001; 32: 1407-9.

4. Kawasaki T. Acute febrile mucocutaneous syndrome with lymphoid involvement with specific desquamation of the fingers and toes in children. Arerugi 1967; 16: 178-222. (Japanese)

5. Kawasaki T, Kosaki F, Okawa S, Shigematsu I, Yanagawa H. A new infantile acute febrile mucocutaneous lymph node syndrome (MLNS) prevailing in Japan. Pediatrics 1974; 54: 271-6.

6. Dadlani GH, Gingell RL, Orie JD, et al. Coronary artery calcifications in the long-term follow-up of Kawasaki disease. Am Heart J 2005; 150: 1016.

7. Kato H, Sugimura T, Akagi T, et al. Long-term consequences of Kawasaki disease. A 10- to 21-year follow-up study of 594 patients. Circulation 1996; 94: 1379-85.

8. Kato H, Akagi T, Sugimura T, et al. Kawasaki disease. Coron Artery Dis 1995; 6: 194-206. (Review)

9. Gersony WM. Diagnosis and management of Kawasaki disease. JAMA 1991; 265: 2699-703. (Review)

10. Newburger JW, Takahashi M, Gerber MA, et al. Diagnosis, treatment, and long-term management of Kawasaki disease: a statement for health professionals from the Committee on Rheumatic Fever, Endocarditis and Kawasaki Disease, Council on Cardiovascular Disease in the Young, American Heart Association. Circulation 2004; 110: 2747-71.

11. Matsuda H, Hashimoto N, Suzuki K, et al. Long-term follow-up of a patient with Kawasaki disease and coronary aneurysm associated with asymptomatic thrombosis: a case report. J Cardiol 2005; 46: 113-8.

12. Hauser M, Bengel F, Kuehn A, et al. Myocardial blood flow and coronary flow reserve in children with "normal" epicardial coronary arteries after the onset of Kawasaki disease assessed by positron emission tomography. Pediatr Cardiol 2004; 25: 108-12.

13. Nakagawa M, Sato A, Okagawa H, Kondo M, Okuno M, Takamatsu T. Detection and evaluation of asymptomatic myocarditis in schoolchildren: report of four cases. Chest 1999; 116: 340-5.

14. Gardner-Medwin JM, Dolezalova P, Cummins C, Southwood TR. Incidence of Henoch-Schonlein purpura, Kawasaki disease, and rare vasculitides in children of different ethnic origins. Lancet 2002; 360: 1197-202.

15. Chang LY, Chang IS, Lu CY, et al. Epidemiologic features of Kawasaki disease in Taiwan, 1996-2002. Pediatrics 2004; 114: e678-82.

16. Chang RK. Hospitalizations for Kawasaki disease among children in the United States, 1988-1997. Pediatrics 2002; 109: e87.

17. Yanagawa H, Nakamura Y, Yashiro M, et al. Incidence survey of Kawasaki disease in 1997 and 1998 in Japan. Pediatrics 2001; 107: E33.

18. Hirata S, Nakamura Y, Matsumoto K, Yanagawa H. Long-term consequences of Kawasaki disease among first-year junior high school students. Arch Pediatr Adolesc Med 2002; 156: 77-80.

19. Ogawa S, Fukazawa R, Ohkubo T, et al. Silent myocardial ischemia in Kawasaki disease: evaluation of percutaneous transluminal coronary angioplasty by dobutamine stress testing. Circulation 1997; 96: 3384-9.

20. Pahl E, Sehgal R, Chrystof D, et al. Feasibility of exercise stress echocardiography for the follow-up of children with coronary involvement secondary to Kawasaki disease. Circulation 1995; 91: 122-8.

21. Chung KJ, Fulton DR, Lapp R, Spector S, Sahn DJ. One-year follow-up of cardiac and coronary artery disease in infants and children with Kawasaki disease. Am Heart J 1998; 115: 1263-7. 